\title{
Métodos de propagação e fatores que interferem na germinação das principais gramíneas nativas de Cerrado
}

\author{
Alexandre Moisés Ericsson de Oliveira ${ }^{1 *}$, Beno Wendling ${ }^{2}$, Débora Baleeiro de Carvalho Ericsson ${ }^{3}$, Mauro \\ Aparecido de Sousa Xavier ${ }^{4}$, Alessandra Rejane Ericsson de Oliveira Xavier ${ }^{5}$
}

\begin{abstract}
Resumo
A propagação via sementes ou vegetativa e a sobrevivência de gramíneas nativas de Cerrado são as etapas mais críticas em um processo de revitalização de áreas degradadas que depende inicialmente da reintrodução dessas espécies no ambiente a ser restaurado e também visando o uso comercial dessas espécies devido à boa palatabilidade e resistência a intempéries ambientais como ausência de chuvas e resistência a praga e doenças de algumas espécies. A falta de estudos sobre a germinação de gramíneas nativas do Cerrado tem sido apontada como importante obstáculo à propagação dessas espécies dificultando a restauração da vegetação nativa do Cerrado. O objetivo deste estudo foi realizar uma revisão sobre as principais gramíneas nativas de cerrado, as formas de propagação utilizadas e fatores que influenciam na sua propagação. O presente estudo seguiu a metodologia de revisão sistemática, a identificação dos artigos foi realizada na base de dados PUBMED www.ncbi.nlm.nih.gov/pubmed/. Devido a rápida devastação do bioma Cerrado com o possível risco de extinção de várias espécies de gramíneas nativas se faz necessário a conservação do germoplasma dessas plantas nativas, além disso, são necessários mais estudos para se avaliar a germinação de gramíneas nativas de cerrado sob diferentes condições abióticas que podem influenciar no desenvolvimento dessas espécies em condições naturais ou próximas, conseguindo assim conhecimento sobre padrões de ocorrência e distribuição das populações, gerando dados essenciais para o entendimento da propagação das espécies e auxiliando principalmente a definir estratégias para a recuperação de áreas degradadas do bioma Cerrado.
\end{abstract}

Palavras-chaves: Conservação do Cerrado. Poaceae. Superação de dormência. Propagação de gramíneas.

\section{Propagation methods and germination interfering factors of the main Cerrado native grasses}

\begin{abstract}
Seed or vegetative propagation and survival of native Cerrado grasses are the most critical steps in a process of revitalization of degraded areas that initially depends on the reintroduction of these species into the environment to be restored and also aiming at the commercial use of these species due to good palatability and resistance to environmental hazards such as rainfall and resistance to pests and diseases of some species. The lack of studies on germination of native Cerrado grasses has been pointed as an important obstacle to the propagation of these species making it difficult to restore native Cerrado vegetation. The aim of this study was to review the main native Cerrado grasses, the forms of propagation used in these grasses and factors that influence the propagation of native Cerrado grasses. The present study followed the systematic review methodology, the articles were identified in the PUBMED database www.ncbi.nlm.nih.gov/pubmed/. Due to the rapid devastation of the Cerrado biome with the possible risk of extinction of several native grass species, it is necessary to preserve the germplasm of these native plants. In addition,
\end{abstract}

${ }^{1}$ Universidade Federal de Uberlândia. Uberlândia, MG. Brasil.

https://orcid.org/0000-0001-8162-9174

${ }^{2}$ Universidade Federal de Uberlândia. Uberlândia, MG. Brasil.

https://orcid.org/0000-0002-8812-1661

${ }^{3}$ Universidade Estadual de Goiás. Pires do Rio, GO. Brasil.

https://orcid.org/0000-0003-2558-8691

${ }^{4}$ Universidade Estadual de Montes Claros. Montes Claros, MG. Brasil.

https://orcid.org/0000-0002-0512-1616

${ }^{5}$ Universidade Estadual de Montes Claros. Montes Claros, MG. Brasil.

https://orcid.org/0000-0001-8558-4196

*Autor para correspondência: moisesericsson@gmail.com

Recebido para publicação em 18 de outubro de 2019. Aceito para publicação em 22 de dezembro de 2019.

e-ISSN: 2447-6218 / ISSN: 2447-6218. Atribuição CC BY. 
Oliveira, A.M.E. et al.

further studies are needed to evaluate the germination of native Cerrado grasses under different abiotic conditions that may influence the development of these species in or near natural conditions, thus obtaining knowledge about patterns of occurrence and distribution of populations, generating essential data for understanding the propagation of species and mainly helping to define strategies for the recovery of degraded areas of the Cerrado biome.

Keywords: Cerrado Conservation. Poaceae. Overcoming of numbness. Grass propagation.

\section{Introdução}

A segunda maior formação vegetal brasileira é o bioma Cerrado, que ocupa aproximadamente 2 milhões de $\mathrm{km}^{2}$ (aproximadamente $23 \%$ da área do Brasil). Seus tipos de vegetação incluem áreas florestais, savana e campestre. O Cerrado foi incluído entre os 34 hotspots do planeta devido ao seu alto grau de endemismo sendo rico biologicamente em uma área ameaçada. Devido a expansão da agricultura no centro do Brasil, nas últimas décadas, o Cerrado sofreu uma rápida redução de sua cobertura vegetal original interferindo na redução da biodiversidade, e invasão por espécies exóticas. A invasão biológica particularmente por gramíneas africanas e mudanças no regime do fogo devido a queimadas não controladas são as maiores ameaças atuais para o bioma Cerrado. Estimativas indicam que cerca da metade da cobertura original do Cerrado foi transformada em pastagens cultivadas, culturas anuais e outros tipos de uso da terra. Atualmente a maior parte do Cerrado apresenta vegetação nativa altamente fragmentada e em muitas áreas onde houve uma mudança de uso da terra para o desenvolvimento das pastagens agropecuárias (Barbosa et al., 2016; Carvalho et al., 2009).

São relatadas cerca de 510 espécies de gramíneas nativas na região do Cerrado, sendo poucas as informações sobre a fenologia reprodutiva, características das sementes, germinação e estabelecimento das espécies de gramíneas nativas de Cerrado, além de outros fatores que podem influenciar o seu estabelecimento no campo, como a fertilidade do solo, chuvas, fogo, competição com outras plantas e herbivoria (Lindsay; Cunningham, 2011; Zaidan; Carreira, 2008). Com isso, torna-se necessário o desenvolvimento de técnicas de manejo que possam ser utilizadas na restauração de áreas de Cerrado degradadas, sendo primordial para sua conservação. Dentre as técnicas utilizadas uma que tem recebido bastante enfoque na recuperação de áreas degradas é a propagação vegetativa, contudo, o seu alto custo, a alta incidência de doenças e pragas devido o uso deste método além da propagação vegetativa ser de difícil aplicação em grandes áreas. Deste modo, é mais viável e prático o uso de sementes utilizando a semeadura direta. (Zahawi; Holl, 2009; Neves et al., 2006; Page; Bork, 2005; Carmona et al., 1999).

Alguns autores afirmam que a falta de conhecimento sobre os meios de propagação de espécies de gramíneas nativas é um dos principais fatores que contribuem para o uso de gramíneas exóticas, como Melinis minutiflora Beauv. e Urochloa spp (Brachiária) para recuperar áreas de Cerrado degradadas, pois suas características são extensivamente conhecidas por serem comumente utilizadas como pastagem (Martins et al., 2011; Silva; Haridasan, 2007). Apesar disso, as principais formas de restauração da composição e abundância de espécies de gramíneas nativas é o uso da propagação vegetativa e plantio com sementes de várias espécies, principalmente com a finalidade da recuperação do estrato rasteiro como forma de diminuir a abundância de espécies exóticas e espécies de gramíneas africanas invasoras. Assim, a caracterização e a investigação dos atributos destas duas formas de propagação de plantas (sementes e vegetativas) são importantes para se entender os processos de estabelecimento e também a competição entre plântulas com a finalidade de produção de mudas, manejo da biodiversidade ou de recuperação de áreas degradadas (Machado et al., 2013; Martins et al., 2011; Foster et al., 2007; Prober et al., 2005).

A falta de estudos sobre a germinação de gramíneas nativas do Cerrado tem sido apontada como importante obstáculo à propagação dessas espécies dificultando a restauração da vegetação nativa do Cerrado. Apesar da dificuldade da propagação destas espécies, existem trabalhos mostrando a necessidade deste tipo de pesquisa para não se perder as centenas de espécies de gramíneas nativas por competição com plantas exóticas ou plantas invasoras e para se conservar o germoplasma principalmente das espécies de gramíneas nativas de cerrado (Martins et al., 2011; Zahawi; Holl, 2009; Zaidan; Carreira, 2008).

O objetivo deste estudo foi realizar uma revisão sistemática sobre as principais gramíneas nativas no bioma Cerrado, visando caracterizar as modalidades de propagação de gramíneas nativas de Cerrado (sementes e vegetativa), abordar as dificuldades das principais técnicas de propagação utilizadas em gramíneas nativas de Cerrado e apontar os principais fatores que afetam a germinação de gramíneas nativas de Cerrado.

O presente estudo seguiu a metodologia de revisão sistemática, o trabalho foi conduzido em etapas que envolveram o desenvolvimento do protocolo de revisão com as questões da pesquisa, a estratégia de busca, a identificação dos critérios de inclusão e exclusão, a busca nas bases de dados previamente definidas, avaliação crítica, extração dos dados relevantes e síntese. A identificação dos artigos foi realizada na base de dados PUBMED www. ncbi.nlm.nih.gov/pubmed/. 
Métodos de propagação e fatores que interferem na germinação das principais gramíneas nativas de Cerrado

Características gerais e principais gramíneas nativas de Cerrado

As gramíneas (família: Poaceae) configuram uma das maiores famílias de angiospermas com uma grande importância ecológica e econômica devido à agricultura e pecuária. São encontradas em praticamente todos os habitats existentes, prevalecendo na vegetação terrestre, cobrindo aproximadamente $30 \%$ da superfície emersa da Terra. O hábito e os ciclos reprodutivos são características morfológicas destas plantas que contribuem para o sucesso da família Poaceae, por exemplo: os meristemas intercalares, bainhas protetoras nas folhas e colmos conferem uma grande tolerância a ataque de insetos, fogo e alagamento. Outras características como a vasta distribuição espacial, ciclos reprodutivos relativamente curtos, produção em massa de sementes e capacidade de reproduzir assexuadamente são fatores dominantes para o estabelecimento desta família de plantas. É interessante ressaltar que as gramíneas são utilizadas pelo homem desde a antiguidade, e atualmente além de grande interesse econômico as gramíneas possuem alta importância ecológica em comunidades naturais, sendo relevante tanto na fixação e estruturação dos solos quanto nas interações com a fauna silvestre (Hodkinson, 2018; Silva; Haridasan, 2007).

Segundo estudo do Centro de Pesquisas Agropecuária do Cerrado (CPAC), da Embrapa, mostrando a detecção de espécies de gramíneas nativas mais selecionadas por bovinos desde a década de 1980, as espécies detectadas foram: Axonopus berbigerus (Kunth) Hitch, A. marginatus (Trin) Chase, Digitaria Sacchariflora (Raddi) Henr, Echinolaena inflexa (Poir) Chase (capim-flechinha), Mesostum loliforme (Hotch) Chase, Paspalum erianthum Ness (capim-branco) (Silva; Almeida, 1986). As principais espécies de gramíneas nativas relatadas encontradas no Cerrado são: Andropogon leucostachyus Kunth, Aristida riparia Trin, Aristida recurvata Kunth, Aristida setifolia Kunth, Aristida torta (Nees) Kunth, Axonopus barbigerus (Kunth) Hitch., Axonpus brasiliensis Kuhlm., Ctenium chapadense (Trin.) Doell, Echinolaena inflexa (Poir.) Chase, Gymnopogon spicatus (Spreng.) Kuntze, Paspalum carinatum Humb., Paspalum gardnerianum Nees, Paspalum reduncum Nees, Paspalum stellatum Humb. e Bonpl. ex Fluggé, Paspalum trachycoleon Steud., Schizachyrium microstachyum (Ham.) Roseng, Thrasya glaziouvii AG Burman e Tristachya leiostachya Nees (Barbosa et al., 2016; Machado et al., 2013; Carmona et al., 1999).

Somente no Distrito Federal foram detectadas 132 espécies de gramíneas nativas, sendo 13 destacadas com alto valor forrageiro, são elas: Actinocladum vericillarum, Agenium goyazense, Arthropogon villosus, Axonopus aureus, Axonopus chrysoblepharis, Axonopus marginarus, Achinolaena inflexa, Mesosetum loliiforme, Paspalum erianthum, Paspahum gardnerianum, Paspalum splendens, Setaria geniculata e Schizachyrium tenerum. Sendo todas estas 13 espécies perenes, encontradas em habitats abertos (campo sujo, campo limpo e brejo) e formações florestais (matas e cerradão). Outras características interessantes são que todas essas 13 espécies são resistentes ao fogo, porém essa resistência não é igual para todas elas (Filgueira, 1992). Já na área do Quadrilátero Ferrífero, localizado na porção sul da Serra do Espinhaço, sendo uma das áreas mais ricas em biodiversidade e espécies endêmicas do Estado de Minas Gerais (Brasil) as principais espécies de gramíneas nativas encontradas são: Andropogon bicornis, Andropogon leucostachyus, Apochloa euprepes, Cyathea brownii, Echinolaena inflexa e Setaria parviflora (Figueiredo et al., 2012).

\section{Métodos de propagação de gramíneas nativas de Cerrado}

As duas principais formas de propagação de gramíneas nativas de cerrado são propagação via sementes e a propagação vegetativa. No entanto, existe uma escassez de informações sobre a germinação de sementes de gramíneas nativas do cerrado, provavelmente devido ao sucesso da propagação vegetativa (Zaidan; Carreira, 2008). Algumas gramíneas nativas de Cerrado têm grande potencial para serem usadas como vegetação pioneira na recuperação de áreas degradadas, como as espécies: Andropogon bicornis L., Andropogon leucostachyus Kunth, Echinolaena inflexa (Poir.) Chase, Setaria parviflora (Poir.) Kerguelen e Apochloa euprepes (Renvoize) Morrone \& Zuloaga, que apresentam como características a morfologia e fisiologia que lhes permitem sobreviver em ambientes hostis, fazendo dessas espécies boas gramíneas para serem utilizadas na reabilitação de áreas de Cerrado (Jacobi et al., 2008). Outra espécie importante é a Cenchrus brownii Roem \& Schult que é uma erva nativa com ampla distribuição nas regiões da América Central e América do Sul, indicando uma boa capacidade de espalhamento em ambientes perturbados (Figueiredo et al., 2012).

\section{Propagação de sementes de gramíneas nativas de Cerrado em laboratório e campo}

Devido as limitações da propagação vegetativa de gramíneas nativas, sempre que possível é preferível se fazer a propagação por sementes. As espécies de gramíneas nativas apresentam várias vantagens em relação a outras espécies introduzidas, como rapidez de desenvolvimento, manutenção da flora e fauna nativas e grande adaptação às condições edafoclimáticas locais. Devido a rápida expansão da agricultura no Cerrado, existe um grande risco de extinção de várias espécies de gramíneas nativas levando a necessidade da conservação desse germoplasma. Com isso estudos sobre as características das sementes de gramíneas nativas são de grande relevância, ajudando a entender suas respostas funcionais às condições ambientais, como também para manejar as diferentes espécies com vistas à sua manutenção e eventual eliminação do pasto nativo (Kolb et al., 2016; Carmona et al., 1999).

A propagação de espécies de gramíneas nativas de cerrado: Andropogon bicornis L., Andropogon leucostachyus 
Oliveira, A.M.E. et al.

Kunth, Echinolaena inflexa (Poir.) Chase, Setaria parviflora (Poir.) Kerguelen e Apochloa euprepes (Renvoize) Morrone \& Zuloaga pode ser feita coletando suas sementes manualmente no campo em ramos que iniciaram a liberação natural de espiguetas. Após a colheita, secá-las à sombra à temperatura ambiente para se realizar o teste de germinação. Espiguetas de Andropogon podem ser selecionadas para a remoção de impurezas sem distinção entre espiguetas vazias e plenas, pois devido ao seu pequeno tamanho, as diferenças entre espiguetas cheias e vazias são mínimas, deixando a seleção manual muito lenta. As espigas de Andropogon podem ser testadas sem remover qualquer estrutura ao redor da cariopse. Já nas espécies E. inflexa e Apochloa euprepes as estruturas em torno da cariopse são removidas esfregando uma pequena quantidade de espigas na palma de uma mão com o polegar da outra mão. Esta mistura de cariopses e palha deve ser homogeneizada em um béquer de 1000 $\mathrm{ml}$ contendo água. Alguns segundos após a homogeneização, o material sobrenadante deve ser descartado e as cariopses no fundo do copo devem ser recolhidas após despejar o conteúdo do copo através de um crivo. Para análise preliminar, dois grupos de cariopses com diferentes densidades devem ser seccionados. A presença de endosperma pode ser observada com uso de lupa. Imediatamente após a seleção de cariopses (sementes) que apresentaram a presença de endosperma, as sementes devem ser levadas para germinar (Carmona et al., 1999; Figueredo et al., 2012).

Para o teste de germinação realizado em laboratório, utiliza-se placas de Petri, colocando cerca de 25 sementes para germinar em duas folhas de papel de filtro. Em cada placa adicionar $4 \mathrm{ml}$ de Nistatina (1000 UI/L) para promover a hidratação das sementes e reduzir a contaminação por fungos. As placas de petri devem ser seladas com fita para evitar a perda de humidade e colocadas numa câmara de germinação a $25^{\circ} \mathrm{C}$ sob iluminação contínua. A posição das placas dentro da câmara deve ser alterada aleatoriamente a cada 24 horas. Para se reduzir a contaminação por microrganismos, especialmente fungos, todas as peças de vidro usadas para germinação de sementes devem ser previamente imersas em solução de ácido clorídrico $(\mathrm{HCl})$ a $1 \%$ por trinta minutos e depois lavadas três vezes com água destilada e secas em estufa a $100^{\circ} \mathrm{C}$. Vários testes podem ser feitos para avaliação de germinação como a germinação na presença de luz, germinação hidratada com solução de nitrato de potássio $\left(\mathrm{KNO}_{3}\right)$ a $0,2 \%$, pré-aquecimento das sementes colocando-as em um forno por dois minutos a $80^{\circ} \mathrm{C}$ sendo depois transferindo as sementes para a câmara de germinação e o uso da escarificação com ácido sulfúrico onde as sementes devem ser imersas em ácido sulfúrico $\left(\mathrm{H}_{2} \mathrm{SO}_{4}\right)$ por três minutos, depois lavadas em água corrente para evitar a contaminação do embrião e transferidas para a câmara de germinação. O uso da escarificação não deve ser feito em sementes de Andropogon, pois devido ao pequeno tamanho da semente, a imersão em ácido pode destruí-las. A germinação deve ser avaliada diariamente, sendo sementes com pelo menos 2-3 mm radícula e/ou coleóptilos sendo considerados como sementes germinadas, contadas e retiradas das placas de petri (Figueiredo et al., 2012; Oliveira; Garcia, 2006).

Trabalhos indicam que espécies de gramíneas nativas, mesmo se nativo de uma área relativamente pequena, respondem de forma muito diferente aos diferentes tratamentos propostos em testes de germinação laboratoriais, reforçando a necessidade de um trabalho mais específico em cada espécie, para se identificar as melhores condições de germinação. Carmona et al. (1999) avaliando sementes de gramíneas nativas, mecanicamente selecionadas e armazenadas por seis meses a temperatura ambiente obtiveram taxa de germinação em laboratório de 27\%. Figueiredo et al. (2012) obtiveram taxas de germinação de $28 \%$ em sementes armazenadas em refrigerador por oito meses a $8^{\circ} \mathrm{C}$ e taxa de germinação de $24 \%$ em tratamentos onde se usou nas sementes solução de $\mathrm{KNO}_{3}$, embora a taxa de germinação encontrada por estes autores seja baixa, estes tratamentos produziram resultados muito melhores do que o resultado da taxa de germinação do controle do tratamento com apenas $18 \%$, além disso esses autores mostraram que o uso de pré-aquecimento em sementes em testes de germinação pode acelerar a germinação das sementes com até $86 \%$ de germinação em uma semana.

A estimulação da germinação de sementes nativas, principalmente das espécies Andropogon bicornis L., Andropogon leucostachyus Kunth, Echinolaena inflexa (Poir.) Chase, Setaria parviflora (Poir.) Kerguelen e Apochloa euprepes (Renvoize) Morrone \& Zuloaga, Cenchrus brownii Roem \& Schult, após o fogo pode ser um resultado da adaptação evolutiva. Após a queima, mais nutrientes estão disponíveis, a competição com outras plantas é menos intensa e os herbívoros são menos ameaçadores, o que pode facilitar o estabelecimento de plântulas que germinam logo após o fogo. É comum se encontrar temperaturas entre $80^{\circ} \mathrm{C} \mathrm{e} 100^{\circ} \mathrm{C}$ a $0,3 \mathrm{~cm}$ e $1 \mathrm{~cm}$ de profundidade na camada superficial do solo sendo observadas em ambientes sazonais de cerrado durante eventos de incêndio. Temperaturas acima de $120^{\circ} \mathrm{C}$, no entanto, são letais para as sementes de gramíneas nativas. Experimentos com semeadura direta em campo, nenhuma semente das espécies Andropogon bicornis, Andropogon leucostachyus Kunth, Axonopus pressus (Steud) Parodi germinou durante o período de observação de três meses, apenas Andropogon leucostachyus que teve a primeira germinação quatro meses e meio após a semeadura (Barbosa et al., 2016; Figueiredo et al., 2012; Lima et al., 2104; Lamont et al., 1993).

Em Andropogon a dormência pode ser superada pelo envelhecimento como pode ser observada em outras gramíneas nativas (Carmona et al., 2015). Estudos mostram que o armazenamento à temperatura ambiente de sementes de Andropogon reduz a taxa de germinação, indicando que o armazenamento sob refrigeração permite a superação da dormência nesta espécie (Figueiredo et 
Métodos de propagação e fatores que interferem na germinação das principais gramíneas nativas de Cerrado

al., 2012). O armazenamento a baixas temperaturas pode reduzir a atividade enzimática, preservando melhor os componentes celulares (Peterbauer; Richter, 2001).

Em um estudo utilizando a gramínea nativa Echinolaena inflexa as sementes coletadas em campo foram armazenadas por seis meses sob uma temperatura constante de $25^{\circ} \mathrm{C}$, usando solo como substrato, neste trabalho o autor obteve $30 \%$ de germinação, que iniciou a partir do sétimo dia do experimento, sugerindo que a superação da dormência de sementes de Echinolaena inflexa é favorecida pelo armazenamento, além de indicar a remoção de estruturas ao redor da cariopse em associação com a germinação sob temperatura alternada de $20-35^{\circ} \mathrm{C}$ e fotoperíodo de 12 horas, o que pode ser positivo na superação da dormência de Echinolaena inflexa (Klink, 1996). Outra boa alternativa às restrições à propagação sexual de Echinolaena inflexa é melhorar sua disseminação com material vegetativo (rizomas) (Marques et al., 2014).

A espécie Apochloa euprepes apresenta taxas de germinação muito baixas em laboratório, não sendo recomendada para uso em recuperação de áreas de cerrado degradadas, alguns trabalhos sugerem que a interrupção da dormência das sementes de A. euprepes, está ligada ao envelhecimento da semente ou devido ao fogo ou a mudanças promovidas pelo fogo como a fumaça. Diversos trabalhos indicam que sementes das principais gramíneas nativas de cerrado como Andropogon bicornis, Andropogon leucostachyus, Setaria parviflora e Cyathea brownii apresentam algum tipo de dormência, sendo o armazenamento refrigerado das sementes por cerca de 8 meses e o pré-aquecimento as melhores formas de quebrar a dormência de algumas espécies como Andropogon e $S$. parviflora, para outras importantes espécies como A. bicornis, $S$. parviflora e $C$. brownii o uso de nitrato de potássio $\mathrm{KNO}_{3}$ aumenta a germinação em laboratório das sementes coletadas após colheita manual mecânica (Carmona et al., 2015; Figueiredo et al., 2012; Fidelis et al., 2007; Carmona et al., 1999, Keith, 1997).

\section{Propagação de sementes de gramíneas nativas de Cerrado em casa de vegetação}

As técnicas de propagação e cultivo de gramíneas nativas de Cerrado dependem do conhecimento da dinâmica germinativa dessas espécies. Com isso é importante se conhecer o comportamento das espécies em diferentes condições abióticas, de forma que a reintrodução seja feita adequadamente de acordo com sua capacidade de sobrevivência e estabelecimento em campo. Estudos em casa de vegetação são interessantes para se conhecer como o grau de cobertura do dossel influencia no estabelecimento e desenvolvimento de plântulas de gramíneas nativas de Cerrado. Esse tipo de estudo além de prover a melhoria das técnicas de produção de mudas ou de propagação dessas espécies por semeadura direta (Cava et al., 2016; Lima et al., 2014; Salazar et al., 2012).
Em um estudo em casa de vegetação verificando a germinação de plantas nativas de cerrado incluindo três gramíneas nativas Andropogon bicornis, Andropogon leucostachyus Kunth, Axonopus pressus (Steud) Parodi, espécies típicas do Cerrado paulista na região de Estação Ecológica de Santa Bárbara (Águas de Santa Bárbara, SP), as sementes coletadas foram comparadas em dois tratamentos: semeadura a pleno sol e sombreamento de $75 \%$ por meio de sombrite. As sementes foram dispostas para germinar em tubetes de polipropileno, preenchidos até a metade com esterco de cavalo curtido, sendo o restante do volume preenchido com terra vegetal. Após a semeadura das sementes coletadas nos tubetes foi feito a avaliação da germinação destas espécies usando como critério para se considerar a semente como germinada, a exposição da parte aérea da plântula na superfície do substrato. Os resultados mostraram que sementes de Andropogon bicornis, tiveram germinação inferior a 10\% além de germinar apenas em ambiente sombreado, já a espécies de Andropogon leucostachyus germinaram apenas em pleno sol, sementes da espécie Axonopus pressus demoraram 121 dias para germinar em ambiente sombreado, já em ambiente com sol a taxa de germinação variou de 90 a 241 dias (Lima et al., 2014; Carvalho et al., 2005).

\section{Propagação vegetativa de gramíneas nativas de Cer- rado}

As gramíneas nativas de cerrado exibem diversas formas de reprodução, bem como sexuada como assexuada (sementes botânicas, rizomas, estolões, caules). É relatado que existem espécies que são propagadas quase que unicamente de forma vegetativa, principalmente pela dificuldade de obtenção de sementes de boa qualidade. É o caso da espécie Paspalum notatum que tem altos índices de esterilidade de espiguetas. A propagação vegetativa por estacas é uma alternativa para a multiplicação de plantas nativas com potencial uso na recuperação de matas ciliares e áreas degradadas, especialmente no caso de espécies com baixo poder germinativo. Esta técnica possibilita a obtenção de material vegetal (mudas vivas) de plantas-mãe próximas ao local de intervenção, mais adaptadas às condições edafoclimáticas locais, a fim de produzir uma grande quantidade de mudas em menor período de tempo e com custos reduzidos. Além disso, o uso de mudas vivas contribui para a estabilização imediata do solo. É recomendado que ao utilizar a propagação vegetativa de espécies nativas para fins ambientais, é essencial que a coleta seja realizada a partir do maior número possível de plantas-mães, a fim de aumentar a variabilidade genética entre os indivíduos gerados (Kettenhuber et al., 2019; Dias et al., 2013; Carmona et al., 1999).

A reprodução por semente de gramíneas nativas como a espécie Andropogon gerardii, ocorre frequentemente sob uma faixa mais estreita de condições ambientais do que a reprodução vegetativa. As principais gramíneas nativas se reproduzem principalmente vegetativamente. Embora essas gramíneas possam apresentar um esforço 
Oliveira, A.M.E. et al.

considerável de floração, o recrutamento de plântulas dessas gramíneas perenes em ambientes não perturbados é raro. Com isso, a maior parte do recrutamento de perfilhos ocorre através da reprodução vegetativa de gemas axilares subterrâneas. Gramíneas perenes dominantes e subdominantes dentro da mesma pastagem podem variar em seu tempo de produção anual de gemas, em seu tamanho e estrutura etária. A reprodução vegetativa de uma gramínea perene pode se alterar próximo ao seu limite de variação, semelhante às mudanças observadas no esforço de floração e produção de sementes de algumas espécies anuais próximas aos seus limites de distribuição. A reprodução vegetativa também pode mudar através dos habitats devido a diferenças na disponibilidade de recursos ou competição (Ott; Hartnett, 2015; Peters, 2000).

Um método para se realizar a propagação vegetativa de gramíneas nativas de cerrado é avaliar se as plantas-mães aparentam ter boas condições fitossanitárias, selecionar plantas com idade e características morfológicas semelhantes. Coletar ramos preferencialmente do último ciclo vegetativo da planta, embalar em sacos plásticos sacos e depois levar para laboratório. As estacas podem ser feitas a partir da parte central do ramo sem folhas usando um corte reto, com um comprimento de $20 \mathrm{~cm}$. As estacas podem ser plantadas na proporção $2 / 3$ enterradas em potes de 1,7 litros preenchidos com areia de peneira média. Este tipo de experimento pode ser conduzido em estufa automatizada a uma temperatura entre $20^{\circ} \mathrm{C}$ e $30^{\circ} \mathrm{C}$ usando irrigação por gotejamento de $10 \mathrm{ml}$ três vezes por dia. Pode ser avaliado a capacidade de propagação vegetativa, desenvolvimento da parte aérea e sistema radicular das espécies (Kettenhuber et al., 2019).

A estaquia que é uma técnica de propagação vegetativa estabelece uma alternativa de suplantação das dificuldades na propagação de espécies de gramíneas nativas de cerrado, abrindo a possibilidade para ser usada também para fins comerciais, assim como amparar no resgate e conservação de áreas degradas do bioma Cerrado. Os pontos negativos da propagação vegetativa são os custos elevados, alta necessidade de mão-de-obra, maior disseminação de doenças e pragas, necessidade de grande quantidade de material propagativo para áreas extensivas, além da mais rápida perecibilidade do material (Carmona et al., 1999).

\section{Fatores que afetam a germinação de sementes nativas de Cerrado}

Estudos apontam que a germinação em espécies nativas do Cerrado é muito variável podendo atingir valores muito diferentes dependendo da espécie avaliada, indo desde a ausência completa de germinação até valores próximos de 100\% de germinação (Lima et al., 2014; Silveira et al., 2013). Fatores ambientais como fogo, luz e altas temperaturas influenciam na germinação de espécies nativas de cerrado. Com isso, é necessário o conhecimento e a identificação de fatores ambientais que podem controlar a germinação de gramíneas nativas do Cerrado, auxiliando para uma melhor compreensão dos processos que regem a regeneração dessas espécies em ecossistemas naturais (Carmona et al., 2015; Le Stradic et al., 2015; Musso et al., 2015).

A baixa porcentagem de germinação em sementes de diferentes espécies nativas de Cerrado pode ser devido a ocorrência de dormência das sementes, mecanismo já relatado para muitas espécies do Cerrado. Para quebrar a dormência dessas sementes para ocorrer a germinação, várias técnicas podem ser utilizadas como o choque térmico, imersão das sementes em ácidos ou outras substâncias químicas, escarificação mecânica ou manual. Em condição ambiental natural, alguns fatores podem estimular a germinação das sementes como: luminosidade, a alternância de temperaturas, passagem das sementes ou dos frutos pelo trato digestivo de certos animais e o fogo (Penfield, 2017; Kolb; et al., 2016; Barbosa et al., 2016; Carmona et al., 2015; Carmona et al., 1999).

A condição e o tempo de armazenamento das sementes são fatores que influenciam na germinação de sementes de gramíneas nativas de Cerrado. Alguns efeitos negativos podem ocorrer devido a estocagem, onde quanto maior o tempo armazenado menor pode ser o poder germinativo. O tempo de armazenamento pode ocasionar problemas como a perda de umidade da semente, resultando em altos níveis de dessecação, com isso as sementes podem reduzir drasticamente sua longevidade e vigor, resultando em redução da germinação (Scalon et al., 2012; Salazar et al., 2011; Rossato; Kolb, 2010). A temperatura também pode influenciar na germinação, no entanto baixas temperaturas de armazenamento podem ser prejudiciais a algumas espécies enquanto outras espécies podem se beneficiar de baixas temperaturas aumentando seu percentual germinativo (Figueiredo et al., 2012).

Outra característica importante é que várias espécies de plantas nativas de Cerrado podem apresentar alta porcentagem de sementes estéreis ou com embriões pouco desenvolvidos, diminuindo ou inibindo a sua germinação. A ampla variação no tempo de germinação nas diferentes espécies de gramíneas de cerrado pode ser uma vantagem evolutiva, aumentando as chances de algumas sementes germinem em condições ambientais mais favoráveis na natureza, principalmente no Cerrado onde se tem um período de seca prolongado e o fogo devido a palha seca formada pela falta de chuvas, porém se pensando em propagação, esse longo intervalo de germinação das mais importantes espécies de gramíneas nativas é um obstáculo pois é mais viável que as sementes de uma mesma espécie germinem simultaneamente, fato que não ocorre na maioria das espécies aqui relatadas (Oliveira et al., 2008).

Alguns trabalhos indicam que a luz é o fator que tem mais influência na germinação de gramíneas 
Métodos de propagação e fatores que interferem na germinação das principais gramíneas nativas de Cerrado

nativas de cerrado, podendo produzir ou não efeitos na germinação dessas plantas e apesar do Cerrado ter como característica luz abundante, as espécies nativas apresentam germinação variada pela disponibilidade de luz (Lima et al., 2014; Rossato; Kolb, 2010; Zaidan; Carreira, 2008). Além disso, alguns autores acreditam que em trabalhos para avaliação de germinação de sementes visando sua propagação para fins de restauração de áreas degradadas, os resultados de trabalhos realizados em casa de vegetação são mais realistas do que trabalhos realizados em laboratório, pois mostram com mais realidade o comportamento das gramíneas tanto na fase inicial da germinação quanto na fase de crescimento inicial, além do que as condições de luz e temperatura são controladas em laboratório distanciando das condições reais submetidas as espécies em característica de ambiente de Cerrado (Lima et al., 2014; Fagundes et al., 2011).

\section{Considerações finais}

São necessários mais estudos executados em viveiro ou em campo para avaliação da taxa de germinação de gramíneas nativas de Cerrado, visando testar a influência diversos fatores abióticos que influenciam no desenvolvimento dessas espécies em condições naturais ou próximas, gerando dados essenciais para o entendimento da propagação destas espécies e ajudando principalmente a definir estratégias para a recuperação de áreas degradadas do bioma Cerrado.

\section{Referências}

Barbosa, E. G.; Pivello, V. R.; Rissi, M. N.; Fidelis, A. 2016. A importância da consideração de espécies invasoras no manejo integrado do fogo. Biodiversidade Brasileira, 6(2): 27-40+. Disponível em: http://www. icmbio.gov.br/revistaeletronica/index.php/BioBR/article/view/522.

Carmona, R.; Martins, C.R.; Fávero, A.P. 2015. Fatores que afetam a germinação de sementes de gramíneas nativas do cerrado. Revista Brasileira de Sementes, 20(1): 16-22. Doi.org/10.17801/0101-3122/ rbs.v20n1p16-22.

Carmona, R.; Martins, C. R.; Fávero, A.P. 1999. Fatores que afetam a germinação de sementes de gramíneas nativas do cerrado. Revista Brasileira de Sementes, 20(1): 16-22, 2015. Doi: 10.17801/01013122/rbs.v20n1p16-22.

Carvalho, F. M. V.; De Marco, P; Ferreira, L. G. 2009. The Cerrado IntoPieces: Habitat Fragmentation as a Function of Landscape Use in the Savannas of Central Brazil. Biological Conservation 142(7): 1392-1403. Doi.org/10.1016/j.biocon.2009.01.031.

Carvalho, M. P.; Santana, D. G.; Ranal, N. A. 2005. Emergência de plântulas de Anacardium humile A. St.-Hil. (Anacardiaceae) avaliada por meio de amostras pequenas. Revista Brasileira de Botânica 28(3): 627-633. Doi.org/10.1590/S0100-84042005000300018.

Cava, M. G. B. C.; Isernhagen, I., Mendonça, A. H.; Durigan, G. 2016. Comparação de Técnicas Para Restauração Da Vegetação Lenhosa De. Hoehnea 43(2): 301-15. Doi.org/10.1590/2236-8906-18/2016.

Chaves, I. S.; Alvarenga, A.A.; Dousseau, S.; Soares, G.C.M.. Souza, E.S. 2011. Germination of Miconia Ligustroides (Melastomataceae) Diaspores Submitted to Different Treatments for Dormancy Overcoming. Revista Brasileira de Botânica 34(3): 335-41. Doi.org/10.1590/s010084042011000300008 .

Dias, P. C.; Oliveira L.S.; Wendling, I. 2013. Estaquia e miniestaquia de espécies florestais lenhosas do Brasil. Pesquisa Florestal Brasileira, 32(72): 453-462. Doi: 10.4336/2012.pfb.32.72.453.

Fagundes, M.; Camargos, M.G.; Da Costa, F.V. 2011. Soil Quality Affects Seed Germination and Seedling Development of Dimorphandra Mollis: Benth. (Leguminosae: Mimosoideae). Acta Botanica Brasilica 25(4): 908-15. Doi.org/10.1590/S0102-33062011000400018.

Filgueira, T.S. Gramíneas forrageiras nativas no Distrito Federal. 1992. Pesquisa Agropecuária Brasileira, 27: 1103-111. Disponível em: https:// seer.sct.embrapa.br/index.php/pab/article/view/3747.
Fidelis, A., S. C. Müller, V. D. P. Pillar, J. P. 2007. Efeito de altas temperaturas na germinação de espécies dos Campos Sulinos. Revista Brasileira de Biociências, 5(2): 354-357. Disponível em: http://www. ufrgs.br/seerbio/ojs/index.php/rbb/article/viewFile/324/283.

Figueiredo, M. A.; Baêta, H. E.; Kozovits, A.R. 2012. Germination of Native Grasses with Potential Application in the Recovery of Degraded Areas in Quadrilátero Ferrífero, Brazil. Biota Neotropica, 12(3): 118-23. Doi.org/10.1590/s1676-06032012000300013.

Foster, B. L.; Cheryl, A. M.; Kane R. K.; Todd, A. As.; Erin, J. Q.; Kindscher, K.. 2007. Restoration of Prairie Community Structure and Ecosystem Function in an Abandoned Hayfield: A Sowing Experiment. Restoration Ecology 15(4): 652-61. Doi.org/10.1111/j.1526-100X.2007.00277.x.

Hodkinson, Trevor R. 2018. Evolution and Taxonomy of the Grasses (Poaceae): A Model Family for the Study of Species-Rich Groups. Annual Plant Reviews, 1. Doi.org/10.1002/9781119312994.apr0622.

Jacobi, C. M.; Flávio F.C.; Vincent, R.C. 2008. Estudo Fitossociológico de Uma Comunidade Vegetal Sobre Canga Como Subsídio Para a Reabilitação de Áreas Mineradas No Quadrilátero Ferrífero, MG. Revista Árvore 32(2): 345-53. Doi.org/10.1590/s0100-67622008000200017

Klink, C. A. 1996. Germination and Seedling Estabilshment of Two Native and One Invading African Grass Species in the Brazilian Cerrado. Journal of Tropical Ecology, 12(1): 139-47. Doi.org/10.1017/ S0266467400009354.

Keith, A. D. A.. 1997. International Association for Ecology Combined Effects of Heat Shock, Smoke and Darkness on Germination of Epacris stuartii Stapf. Oecologia 112 (3): 340-44.

Kettenhuber, P. W.; Sousa, R.; Sutili, F.. 2019. Vegetative Propagation of Brazilian Native Species for Restoration of Degraded Areas. Floresta e Ambiente 26(2). Doi.org/10.1590/2179-8087.095617.

Kolb, R. M.; Pilon, N.A.L.; Durigan, G. 2016. Factors Influencing Seed Germination in Cerrado Grasses. Acta Botanica Brasilica 30(1): 87-92. Doi.org/10.1590/0102-33062015abb0199.

Lamont, B.B. 1993. Post-Fire Litter Microsites : Safe for Seeds, Unsafe for Seedlings. Ecological Society of America, 74(2): 501-512. Doi: $10.2307 / 1939311$.

Le stradic, S.; Silveira, F.A.O; Buison, E. Diversity of germination strategies and seed dormancy in herbaceous species of campo rupestre grasslands. Austral Ecology, 40(5): 537-546, 2015. Doi.org/10.1111/ aec. 12221 . 
Oliveira, A.M.E. et al.

Lima, Y. B. C.; Durigan, G.; Souza, F.M. 2014. Germinação de 15 Espécies Vegetais Do Cerrado Sob Diferentes Condições de Luz. Bioscience Journal, 30(6): 1864-72. Disponível em: http://www.seer.ufu.br/ index.php/biosciencejournal/article/view/23274.

Lindsay, E. A., Cunningham, S.A. 2011. Native Grass Establishment in Grassy Woodlands with Nutrient Enriched Soil and Exotic Grass Invasion. Restoration Ecology 19: 131-40. Doi.org/10.1111/j.1526100X.2010.00680.x.

Machado, V.M.; Santos, J.B.; Pereira, I.M.; Lara, R.O.; Cabral, C.M.; Amaral, C.S. 2013. Avaliação Do Banco de Sementes de Uma Área Em Processo de Recuperação Em Cerrado Campestre. Planta Daninha 31(2): 303-12. Doi.org/10.1590/s0100-83582013000200007.

Marques, T.D.; Baêta, H.E.; Leita, M.G.P.; Martins, S.V.; Kozovits, A.R. 2014. Crescimento de espécies nativas de cerrado e de Vetiveria zizanioides em processos de revegetação de voçorocas. Ciência Florestal, 24(4): 843-855. Doi.org/10.5902/1980509816584.

Martins, C. R.; Hay, J.V.; Walter, B.M.T.; Proença, C.E.B.; Vivaldi, L.J. 2011. Impact of Invasion and Management of Molasses Grass (Melinis Minutiflora) on the Native Vegetation of the Brazilian Savanna. Revista Brasileira de Botanica 34(1): 73-90. Doi.org/10.1590/S010084042011000100008 .

Musso, C.; Miranda, H.S.; Aires, S.S.; Bastos, A.C.; Soares, A.M.V.; Loureiro, S. 2015. Simulated Post-Fire Temperature Affects Germination of Native and Invasive Grasses in Cerrado (Brazilian Savanna). Plant Ecology and Diversity 8(2): 219-27. Doi.org/10.108 0/17550874.2014.910714.

Neves, T. S.; Carpanezzi, A.A.; Zuffellato-Ribas, K.C.; Marenco, R.A. 2006. Enraizamento de Corticeira-Da-Serra Em Função Do Tipo de Estaca e Variações Sazonais. Pesquisa Agropecuaria Brasileira 41(12): 1699-1705. Doi.org/10.1590/S0100-204X2006001200003.

Oliveira, A.K.M.; Scheleder, E.J.; Favero, S. 2008. Morphological characterization, viability and vigor of Tabebuia Chrysotricha (Mart . Ex. DC .) Standl SeedS. Revista Árvore, 32(6): 1011-18. Doi.org/10.1590/ S0100-67622008000600006.

Oliveira, P.G.; Garcia, Q. S. 2006. Efeitos Da Luz e da Temperatura na Germinação de Sementes de Syngonanthus Elegantulus Ruhland, S. Elegans (Bong.) Ruhland e S. Venustus Silveira (Eriocaulaceae). Acta Botanica Brasilica, 19(3): 639-45. Doi.org/10.1590/s010233062005000300026 .

Ott, J.P; Hartnett, D.C. 2015. Vegetative Reproduction and Bud Bank Dynamics of the Perennial Grass Andropogon Gerardii in Mixedgrass and Tallgrass Prairie. The American Midland Naturalist, 174(1): 14-32. Doi.org/10.1674/0003-0031-174.1.14.

Page, H.N.; Bork, E.W. 2005. Effect of Planting Season, Bunchgrass Species, and Neighbor Control on the Success of Transplants for Grassland Restoration. Restoration Ecology, 13(4): 651-58. Doi. org/10.1111/j.1526-100X.2005.00083.x.

Penfield, Steven. 2017. Seed Dormancy and Germination. Current Biology 27 (17): R874-78. Doi.org/10.1016/j.cub.2017.05.050.
Peterbauer, T.; Richter, A. 2001. Biochemistry and physiology of raffinose family oligosaccharides and galactosyl cyclitols in seeds. Seed Science Research, 11: 185-197. Doi.org/10.1079/SSR200175.

Peters, D.P.C. 2000. Climatic Variation and Simulated Patterns in Seedling Establishment of Two Dominant Grasses at a Semi-Arid-Arid Grassland Ecotone." Journal of Vegetation Science 11(4): 493-504. Doi.org/10.2307/3246579.

Prober, S. M.; Thiele, K.R.; Lunt, I.D.; Koen, T.B. 2005. Restoring Ecological Function in Temperate Grassy Woodlands: Manipulating Soil Nutrients, Exotic Annuals and Native Perennial Grasses through Carbon Supplements and Spring Burns. Journal of Applied Ecology 42(6): 1073-85. Doi.org/10.1111/j.1365-2664.2005.01095.x.

Rossato, D.R.; Kolb, R.M. 2010. Germinação de Pyrostegia venusta (Bignoniaceae), viabilidade de sementes e desenvolvimento pósseminal Brazilian Journal of Botany, 2: 51-60. Doi.org/10.1590/ S0100-84042010000100006.

Salazar, A.; Goldstein, G.; Franco, A.C.; Miralles-Wilhelm, F. 2011. Timing of Seed Dispersal and Dormancy, Rather than Persistent Soil SeedBanks, Control Seedling Recruitment of Woody Plants in Neotropical Savannas. Seed Science Research 21(2): 103-16. Doi.org/10.1017/ S0960258510000413.

Salazar, A.; Goldstein, G.; Franco, A.C.; Miralles-Wilhelm, F. 2012. Seed limitation of woody plants in Neotropical savannas. Plant Ecology, 213(2): 273-287. Doi.org/10.1007/s11258-011-9973-4.

Scalon, S. P. Q.; Neves, E.M.S.; Maseto, T.E.; Pereira, Z.V. 2012. Sensibilidade à Dessecação e Aoarmazenamento Em Sementes de Eugenia Pyriformis Cambess. (Uvaia). Revista Brasileira de Fruticultura 34(1): 269-76. Doi.org/10.1590/s0100-29452012000100036.

Silva, J. S. O.; Haridasan, M. 2007. Acúmulo de Biomassa Aérea e Concentração de Nutrientes Em Melinis Minutiflora P. Beauv. e Gramíneas Nativas Do Cerrado. Revista Brasileira de Botânica 30(2): 337-44. Doi. org/10.1590/s0100-84042007000200016.

Silva, J.C.S.; Almeida, S.P. 1986. Germinação de Gramíneas Nativas dos Cerrados. Centro de Pesquisas Agropecuária do Cerrado (CPAC), 18.

Silveira, C.E.S.; Palhares, D.; Pereira, L.A.R.; Pereira, K.B.D.; Silva,F.A.B. 2013. Strategies of Plant Establishment of Two Cerrado Species: Byrsonima Basiloba Juss. (Malpighiaceae) and Eugenia Dysenterica Mart. Ex DC (Myrtaceae). Plant Species Biology 28(2): 130-37. Doi. org/10.1111/j.1442-1984.2012.00366.x.

Zahawi, R. A.; Holl, K.D. 2009. Comparing the Performance of Tree Stakes and Seedlings to Restore Abandoned Tropical Pastures. Restoration Ecology 17 (6): 854-64. Doi.org/10.1111/j.1526-100X.2008.00423.x.

Zaidan, L.B.P; Carreira, R.C. 2008. Seed Germination in Cerrado Species. Brazilian Journal of Plant Physiology, 20(3): 167-81. Doi.org/10.1590/ S1677-04202008000300002. 